\title{
A FAMÍlIA NO CUIDADO DO PACIENTE COM DIABETES MELLITUS: REVISÃO INTEGRATIVA DA LITERATURA
}

\author{
THE FAMILY IN THE CARE OF PATIENTS WITH DIABETES MELLITUS: \\ AN INTEGRATIVE LITERATURE REVIEW
}

\section{Mayanne Santana de Araújo', Diana Magna Fonseca da Cruz', Patrícia Mariz de Medeiros¹, Pétala Tuani Candido de Oliveira Salvador', Viviane Euzébia Pereira Santos ${ }^{1}$}

${ }^{1}$ Grupo de Pesquisa do Laboratório de Investigação do Cuidado, Segurança e Tecnologias em Saúde e Enfermagem da Universidade Federal do Rio Grande do Norte - Natal (RN), Brasil.

Data de entrada do artigo: 02/01/2013

Data de aceite do artigo: 06/05/2013

\section{RESUMO}

Introdução: A diabetes mellitus (DM) é uma doença crônica que tem sido diagnosticada em um número elevado de indivíduos. Caracteriza-se por deficiência metabólica, que resulta em hiperglicemia crônica. No cuidado em saúde, é importante considerar medidas terapêuticas, as quais abrangem relações existentes no contexto sociocultural, envolvendo o paciente, o profissional de saúde e os familiares. Esse cuidado é determinado pelo conjunto de medidas, as quais podem ser propostas por tais membros, que recuperam a razão de viver de um ser humano. Objetivo: Descrever o conhecimento nacional produzido sobre o processo de cuidar de familiares de pessoas com complicações por diabetes mellitus. Materiais e Métodos: Trata-se de uma revisão integrativa da literatura sobre o desenvolvimento do processo de cuidar de familiares de pessoas com complicações pela diabetes mellitus e as estratégias de cuidado utilizadas pelos familiares. Inicialmente, foi encontrado um total de 473 artigos e, seguindo os critérios de inclusão e exclusão, o número foi reduzido a 12 artigos. Esses foram analisados segundo o ano, o desenho metodológico e a temática abordada. Conclusão: Ressaltou-se a importância da presença do familiar no processo de cuidar dos portadores de diabetes mellitus.

Palavras-chave: diabetes mellitus; cuidado; família; Enfermagem.

\section{ABSTRACT}

Introduction: Diabetes mellitus (DM) is a chronic disease that has been diagnosed in a large number of individuals. It is characterized by metabolic deficiency that results in chronic hyperglycemia. In health care, it is important to consider therapeutic measures, which involve relationships within the sociocultural context, as the patient, health professionals and family members. This care is determined by the set of measures, that may be proposed by these members, which recover the reason to live to a human. Objective: To describe the national knowledge produced about the process of caring for family members of people with complications from diabetes mellitus. Materials and Methods: It is an integrative review of the literature on the development of the process of caring for family members of people with complications from diabetes and the care strategies used by family members. Initially, it was found a total of 473 articles and, following the inclusion and exclusion criteria, the number was reduced to 12 articles. These were analyzed according to year, methodological design and theme. Conclusion: It was emphasized the importance of the presence of the family in the care process along with diabetes mellitus.

Keywords: diabetes mellitus; care; family; Nursing. 


\section{INTRODUÇÃO}

Segundo a Biblioteca Virtual em Saúde (BVS), por meio dos Descritores em Ciências da Saúde (DeCS), o descritor "diabetes mellitus" significa "grupo de transtornos heterogêneos caracterizados por hiperglicemia e intolerância à glicose", e o descritor "família" refere-se ao "grupo social que consiste de pais ou pais substitutos e crianças" 1 .

A diabetes mellitus (DM) pertence a um grupo de doenças crônicas que tem sido diagnosticada em um número elevado de indivíduos. Ela se caracteriza por uma deficiência metabólica, que resulta hiperglicemia crônica. A enfermidade tem algumas classificações, de acordo com sinais, sintomas e a ocasião em que foi diagnosticada, como é o caso da diabetes gestacional ${ }^{2}$.

A doença pode ocasionar complicações sistêmicas em alguns órgãos, dentre eles olhos, rins, nervos, cérebro, coração e vasos sanguíneos ${ }^{3}$. O principal motivo para que ocorram as complicações diabéticas são os hábitos de vida pouco saudáveis que tem o sujeito portador de $\mathrm{DM}^{2}$.

Nessa perspectiva, a transformação do modo de pensar, agir e viver do paciente pode livrá-lo de sérios problemas de saúde. Desse modo, com o aparecimento das manifestações físicas, o paciente inicia a busca por cuidados e tratamento. Nesse momento, é importante a presença do familiar que passa a conviver e cuidar de um membro familiar diabético. Para melhor eficácia, é essencial o compartilhamento dos conhecimentos de todos os componentes do processo de cuidar - profissional, paciente e familiar. Em outras palavras, apreende-se que a integralidade do cuidado possibilita trocas de conhecimentos e melhoria no processo do cuidar ${ }^{4,5}$

No cuidado em saúde é importante considerar medidas terapêuticas que se pautem nas relações existentes no contexto sociocultural, que envolve o paciente, o profissional de saúde e os familiares. Esse cuidado, nesse ínterim, é determinado pelo conjunto de medidas que podem ser propostas por tais membros, os quais geram, organizam e recuperam a razão de viver de um ser humano, que é extremamente capaz de tomar suas próprias decisões e perceber seus limites.

Diante das discussões atuais acerca da diabetes mellitus, de seu processo de cuidar e da essencialidade da família nesse panorama para a valorização da integralidade de todos os envolvidos (profissional, paciente e familiares), e seguindo a configuração da Enfermagem enquanto profissão qualificada do cuidar, apresentamse como questões norteadoras deste estudo: como se desenvolve o processo de cuidar de familiares de pessoas com complicações pela diabetes? Quais estratégias de cuidado são utilizadas pelos familiares?
Objetivou-se, assim, descrever o conhecimento nacional produzido sobre o processo de cuidar de familiares de pessoas com complicações por diabetes mellitus.

\section{MÉTODOS}

Trata-se de uma revisão integrativa da literatura sobre a família no cuidado do paciente com diabetes mellitus e as estratégias utilizadas pelos familiares. A revisão integrativa examina as pesquisas que são pertinentes para a tomada de decisão e o benefício da prática clínica, gerando, consequentemente, um saber a respeito de um tema definido, além de apontar lacunas de conhecimento, o que possibilita o preenchimento delas com novos estudos ${ }^{6}$.

Visando à busca de múltiplos estudos para que se obtivesse um maior leque de conhecimento sobre um assunto em particular ${ }^{6}$, utilizaram-se três descritores, sendo um não controlado - cuidado - e dois controlados - família e diabetes mellitus. Utilizaram-se para a coleta duas combinações "diabetes mellitus e cuidado" e "diabetes mellitus e família" em duas bases de dados científicos - LILACS e BDENF.

Estabeleceu-se como critérios de inclusão: artigos, disponíveis eletronicamente nas bases de dados citadas, que versassem sobre a temática estudada, disponíveis em texto completo, realizados com pacientes adultos $\mathrm{e}$ publicados no idioma nacional.

A coleta de dados foi realizada em julho de 2012 , em pares. Seguindo protocolo de pesquisa, os estudos foram pré-selecionados e então analisados, buscando-se responder aos seguintes indicadores de coleta: ano de publicação, desenho metodológico e temática abordada.

\section{DESENVOLVIMENTO}

De acordo com o protocolo de pesquisa, os dados foram analisados e proporcionaram o desenvolvimento e a apresentação dos resultados. Elucidaram-se, primeiramente, os resultados quantitativos, segundo as combinações utilizadas na coleta de dados (Tabela 1).

Tabela 1: Quantitativo de estudos encontrados e préselecionados, em número absoluto, 2012.

\begin{tabular}{lcc}
\hline Combinação & $\begin{array}{c}\text { Resultado } \\
\text { da pesquisa }\end{array}$ & $\begin{array}{c}\text { Estudos pré-selecionados } \\
\text { conforme critérios } \\
\text { de inclusão }\end{array}$ \\
\hline $\begin{array}{l}\text { Diabetes mellitus } \\
\text { e cuidado }\end{array}$ & 229 & 15 \\
\hline $\begin{array}{l}\text { Diabetes mellitus } \\
\text { e família }\end{array}$ & 244 & 24 \\
\hline Total & 473 & 39 \\
\hline
\end{tabular}


A primeira combinação trouxe um quantitativo de $229(48,4 \%)$ estudos, enquanto a segunda combinação abrangeu um total de 244 (51,6 \%) artigos, gerando um total de $473(100 \%)$ estudos encontrados a priori.

Esse total, conforme análise dos critérios de inclusão, se reduziu a 39 artigos, o que corresponde a $8,2 \%$ do número inicial. Em um segundo momento, os dados foram analisados de acordo com os indicadores da pesquisa, verificando-se a pertinência dos estudos para responder às questões de pesquisa estabelecidas. Nessa etapa, foi possível definir a amostra real da revisão literária: 13 estudos, os quais estão resumidamente expostos na Tabela 2.

Os trabalhos incluídos englobaram: um estudo qualitativo assistencial $(7,70 \%)$, um transversal $(7,70 \%)$, um descritivo qualitativo $(7,70 \%)$, duas pesquisas exploratórias $(15,37 \%)$, duas entrevistas $(15,37 \%)$, uma pesquisa documental $(7,70 \%)$, um estudo epidemiológico $(7,70 \%)$, duas pesquisas qualitativas $(15,37 \%)$ e dois estudos de casos (15,37\%). A dimensão temporal das publicações variou entre 2007 e 2011, com maior incidência de publicações no ano 2009 e 2010, com quatro artigos $(30,76 \%)$.

$\mathrm{Na}$ sociedade atual, a alimentação acontece de maneira rápida e prática, e a evolução tecnológica diminui o esforço físico das atividades diárias, o que propicia elevado número de fatores de risco para doenças crônicas, como o sedentarismo e o sobrepeso, todos passados de pai para filhos ${ }^{7}$.

Com isso, doenças crônicas, antes consideradas fora do comum, eclodem em maiores proporções a cada dia, o que eleva a morbimortalidade da população e se torna um indicativo importante sobre a qualidade de vida desta ${ }^{8}$.

A diabetes mellitus é uma doença que necessita de mudanças de hábitos de vida, sendo imperativo que o portador dela tenha conhecimento sobre a doença,

Tabela 2: Síntese dos estudos incluídos na revisão literária, 2012.

\begin{tabular}{|c|c|c|c|}
\hline Título do estudo & Ano & Desenho metodológico & Temática abordada \\
\hline $\begin{array}{l}\text { Itinerário terapêutico do adolescente com } \\
\text { DM } 1 \text { e seus familiares }\end{array}$ & 2007 & $\begin{array}{l}\text { Qualitativo, convergente } \\
\text { e assistencial. }\end{array}$ & $\begin{array}{l}\text { Itinerário terapêutico de adolescente com DM e } \\
\text { seus familiares. }\end{array}$ \\
\hline $\begin{array}{l}\text { Conhecimento sobre DM de pacientes } \\
\text { atendidos em programa ambulatorial } \\
\text { interdisciplinar de um hospital universitário }\end{array}$ & 2008 & $\begin{array}{l}\text { Estudo descritivo com } \\
\text { abordagem qualitativa }\end{array}$ & $\begin{array}{l}\text { Conhecimentos adquiridos por diabéticos } \\
\text { acerca da DM. }\end{array}$ \\
\hline $\begin{array}{l}\text { Vivências em família das necessidades } \\
\text { de cuidados referente à insulinoterapia e } \\
\text { prevenção de pé diabético }\end{array}$ & 2008 & $\begin{array}{l}\text { Entrevista (descritivo } \\
\text { exploratório) }\end{array}$ & $\begin{array}{l}\text { Práticas de cuidados adotadas por familiares } \\
\text { de pessoas diabéticas. }\end{array}$ \\
\hline $\begin{array}{l}\text { O cuidado à pessoa diabética e as } \\
\text { repercussões na família }\end{array}$ & 2008 & Entrevista (qualitativo) & $\begin{array}{l}\text { Repercussões familiares da assistência oferecida } \\
\text { após um programa educativo sobre diabetes. }\end{array}$ \\
\hline $\begin{array}{l}\text { Diabetes mellitus juvenil: a experiência de } \\
\text { familiares de adolescentes e } \\
\text { pré-adolescentes }\end{array}$ & 2009 & Entrevista (qualitativo) & $\begin{array}{l}\text { Experiência de familiares no cuidar de } \\
\text { adolescentes e pré-adolescentes com DM }\end{array}$ \\
\hline $\begin{array}{l}\text { A experiência em família de uma pessoa } \\
\text { com diabetes mellitus e em hemodiálise }\end{array}$ & 2009 & $\begin{array}{l}\text { Estudo de caso } \\
\text { (qualitativo) }\end{array}$ & $\begin{array}{l}\text { Experiência familiar de uma pessoa diabética e } \\
\text { com insuficiência renal crônica. }\end{array}$ \\
\hline $\begin{array}{l}\text { Diabetes mellitus na adolescência: } \\
\text { experiências e sentimentos dos } \\
\text { adolescentes e da mãe com a doença }\end{array}$ & 2009 & $\begin{array}{l}\text { Pesquisa qualitativa, } \\
\text { reflexiva }\end{array}$ & $\begin{array}{l}\text { Experiências, sentimentos, tratamento e } \\
\text { cuidados de saúde, sobre adolescentes e mãe. }\end{array}$ \\
\hline $\begin{array}{l}\text { A vida cotidiana de quem vivencia a } \\
\text { condição crônica da diabetes mellitus }\end{array}$ & 2009 & $\begin{array}{l}\text { Estudo de caso } \\
\text { (qualitativo) }\end{array}$ & $\begin{array}{l}\text { Experiência de adoecimento de uma pessoa e } \\
\text { condição crônica da DM. }\end{array}$ \\
\hline $\begin{array}{l}\text { Diabetes e suas comorbidades no } \\
\text { Programa Saúde da Família de Vila Davi } \\
\text { em Bragança paulista/SP }\end{array}$ & 2010 & $\begin{array}{l}\text { Estudo epidemiológico, } \\
\text { descritivo. }\end{array}$ & Pacientes diabéticos e suas comorbidades. \\
\hline $\begin{array}{l}\text { Estratégia Saúde da Família no tratamento } \\
\text { de doenças crônico-degenerativas: avanços } \\
\text { e desafios }\end{array}$ & 2010 & $\begin{array}{l}\text { Estudo retrospectivo, } \\
\text { exploratório e } \\
\text { quantitativo }\end{array}$ & $\begin{array}{l}\text { Efetividade da Estratégia Saúde da Família no } \\
\text { tratamento crônico-degenerativo. }\end{array}$ \\
\hline $\begin{array}{l}\text { Fatores de risco cardiovasculares em } \\
\text { adultos jovens com hipertensão arterial e } \\
\text { diabetes mellitus. }\end{array}$ & 2010 & $\begin{array}{l}\text { Estudo documental, } \\
\text { descritivo }\end{array}$ & $\begin{array}{l}\text { Fatores de risco em adultos jovens com } \\
\text { hipertensão arterial e diabetes mellitus. }\end{array}$ \\
\hline $\begin{array}{l}\text { Acesso a serviços de saúde na Baixada } \\
\text { Santista de pessoas portadoras de } \\
\text { hipertensão arterial e/ ou diabetes mellitus }\end{array}$ & 2010 & Estudo transversal & $\begin{array}{l}\text { Acesso do paciente portador de hipertensão } \\
\text { arterial e/ ou diabetes mellitus à rede de saúde } \\
\text { e medicamentos }\end{array}$ \\
\hline $\begin{array}{l}\text { Mulheres com diabetes mellitus } \\
\text { gestacional: conhecendo a doença e } \\
\text { convivendo com ela }\end{array}$ & 2011 & $\begin{array}{l}\text { Pesquisa qualitativa, } \\
\text { descritivo exploratório }\end{array}$ & $\begin{array}{l}\text { Conhecimento das gestantes sobre a diabetes } \\
\text { mellitus e sua vivência com essa. }\end{array}$ \\
\hline
\end{tabular}


terapia medicamentosa, nutrição e mudanças em suas atividades diárias (exercício físico), automonitorização (nível glicêmico), cuidados com os pés e demais partes do corpo, e conscientização de todas as mudanças, estabelecendo resultados no cuidado ${ }^{9}$.

A doença crônica traz consigo a necessidade ímpar de se repensar a maneira como ela incide e afeta a vida da pessoa e de sua família, ocasionando diversas mudanças cotidianas. Isso implica que os profissionais possam reconsiderar o modo de organização das práticas de atenção e de gestão em saúde.

Em outras palavras, ressalta-se que as necessidades de saúde das pessoas que convivem com a condição crônica da diabetes mellitus precisam ser apreendidas e compreendidas em seu cotidiano, com as pessoas que estão presentes em sua vivência, pois é nesse espaço próprio que elas enfrentam suas dificuldades, bem como podem encontrar a maneira de resolvê-las ${ }^{10}$.

A medida terapêutica, nessa perspectiva, inicia-se com a percepção das manifestações físicas que interferem no cotidiano do paciente. Nesse momento, paciente e família começam a busca por cuidados e tratamentos que combatam essas manifestações. Assim, é importante que o profissional construa um vínculo com os pacientes e familiares para que estes entendam melhor o que as pessoas fazem na busca por tratamento, como também porque o fazem e como se mostram abertos e dispostos a estabelecer novas trocas de conhecimentos e acentuar o respeito ${ }^{5}$.

O vínculo estabelecido pelos profissionais com o paciente e seus familiares possibilita a ampliação de conhecimento para ambos os envolvidos nesse processo de cuidar acerca do diabetes no contexto familiar. O paciente, nessa conjuntura, é considerado um elemento importante, sendo ele capaz de promover a união entre os profissionais e familiares, facilitando a edificação do tratamento e a adição de hábitos mais saudáveis em seu dia a dia ${ }^{5}$.

É essencial, nesse ínterim, a consolidação da educação em saúde, não apenas para profissionais mas também para pacientes e seus familiares, tornando o processo de cuidar mais efetivo ${ }^{5}$.

Outra maneira terapêutica importante é a análise psicossocial do portador de diabetes e de seus familiares. Nesse contexto, o profissional deve estar atento às mudanças de comportamento, já que a diabetes em condição crônica, com suas alterações de estilo de vida, pode trazer para o ser humano sentimentos agressivos e de revolta. E, nesse processo, o familiar tem mais facilidade de atuar e contribuir para que esses sentimentos sejam revertidos em disposição para cuidar e tratar da doença, já que o contato próximo está evidente ${ }^{11}$.

Em síntese, apreende-se que a doença crônicodegenerativa necessita que o portador desenvolva o autocuidado, com participação da sua família. Mesmo com atendimento de profissionais, o maior responsável pelo tratamento será o indivíduo e sua família, tornando o paciente eficiente nas informações adquiridas pela equipe de saúde, constituindo um melhor controle da doença ${ }^{12}$.

\section{CONSIDERAÇÕES FINAIS}

A revisão literária colocou em relevo aspectos essenciais do conhecimento sobre a diabetes mellitus, sobretudo acerca da importância dos familiares para o desenvolvimento efetivo do processo de cuidar. Nesse sentido, realça-se que a integralidade entre profissional, paciente e familiar possibilita excelentes resultados na aceitação de mudança de vida e combate à doença.

Em relação às questão norteadoras - como se desenvolve o processo de cuidar de familiares de pessoas com complicações pela diabetes e quais estratégias de cuidado são utilizadas pelos familiares? - , pode-se afirmar que foi possível ter uma visão desse processo de cuidar em que os familiares têm a capacidade de emergir ao paciente e compreender as diversas maneiras de cuidar existentes para o tratamento da doença.

Desse modo, evidencia-se que o processo de cuidar inicia-se com a busca por conhecimentos, a fim de se escolher o tratamento adequado e eficaz. Esse conhecimento proporciona trocas entre profissionais, pacientes e familiares, tendo em vista as condições de vida do paciente. Nesse panorama, o familiar é o elemento principal para concretizar as mudanças de hábitos necessárias aos pacientes no tratamento da doença.

Portanto, o familiar tem grande importância no processo de cuidar do paciente portador de doença crônica, o que pode garantir sentimentos e práticas em saúde favoráveis à adoção de novo estilo de vida e tratamento, acumulando saúde ao paciente.

Espera-se, a partir das reflexões tecidas, contribuir para que a inserção do familiar do sujeito acometido com diabetes mellitus em seu processo de cuidar seja solidificada, tendo em vista o papel fundamental da família na promoção integral da saúde desse indivíduo. 


\section{REFERÊNCIAS}

1. Biblioteca Virtual em Saúde. Descritores em Ciências da Saúde. 2012. [acesso em: 17 jul 2012]. Disponível em: http://decs.bvs.br/

2. Morais GFC, Soares MJGO, Costa MML, Santos IBC. O diabético diante do tratamento, fatores de risco e complicações crônicas. Rev Enferm UERJ. 2009 abr/jun; 17(2):240-5.

3. Brasil. Ministério da Saúde. Cadernos de Atenção Básica, n. 16. Brasília: Ministério da Saúde; 2006. [acesso em: 17 jul 2012]. Disponível em: http://bvsms.saude.gov.br/bvs/ publicacoes/diabetes_mellitus.pdf

4. Mattosinho MM, Silva DM. Itinerário terapêutico do adolescente com diabetes mellitus tipo 1 e seus familiares Rev Latino-am Enferm [periódico on-line]. 2007 nov/dez; 15(6). [acesso em: 17 jul 2012]. Disponível em: http://www. scielo.br/pdf/rlae/v15n6/pt_08.pdf

5. Zanetti ML, Biagg MV, Santos MA, Péres DS, Teixeira CR. O cuidado à pessoa diabética e as repercussões na família. Rev Bras Enferm. 2008 mar/abr; 61(2):186-92.

6. Mendes KDS, Silveira RCCP, Galvão CM. Revisão integrativa: método de pesquisa para a incorporação de evidências na saúde e na enfermagem. Texto Contexto Enferm. 2008 out/dez; 17(4):758-64.
7. Moreira TMM, Gomes EB, Santos JC. Fatores de risco cardiovasculares em adultos jovens com hipertensão arterial e/ou diabetes mellitus. Rev Gaúcha Enferm. 2010 dez; 31(4):662-9.

8. Viggiano CE. Uma revisão sobre diabetes melito. Revista Brasileira de Ciências da Saúde. 2007 jan/mar; 5(11):52-62.

9. Gil GP, Haddad MC, Guariente MH. Conhecimento sobre diabetes mellitus de pacientes atendidos em programa ambulatorial interdisciplinar de um hospital universitário público. Semina Cienc Biol Saúde. 2008 jul/dez; 29(2):141-54.

10. Faria AP, Bellato R. A vida cotidiana de quem vivencia a condição crônica do diabetes mellitus. Rev Esc Enferm USP. 2009 dez; 43(4):752-9.

11. Almino MA, Queiroz MV, Jorge MS. Diabetes mellitus na adolescência: experiências e sentimentos dos adolescentes e das mães com a doença. Rev Esc Enferm USP. 2009 dez; 43(4):750-7.

12. Zavatini MA, Cuman KN, Obreli-neto PR. Estratégia saúde da família no tratamento de doenças crônicodegenerativas: avanços e desafios. Rev Gaúcha Enferm. 2010 dez; 31(4):647-54.

\section{Endereços para correspondência:}

Mayanne Santana de Araújo

mayanne_meyne@hotmail.com

Diana Magna Fonseca da Cruz

dianamagna@hotmail.com

Patrícia Mariz de Medeiros

pattymmariz@hotmail.com
Pétala Tuani Candido de Oliveira Salvador

petalatuani@hotmail.com

Viviane Euzébia Pereira Santos

vivianeepsantos@gmail.com 